\title{
Real-Time Prediction of Electricity Distribution Network Status Using Artificial Neural Network Model: A Case Study in Salihli (Manisa, Turkey)
}

\author{
Mahmut Sayar ${ }^{1 *}$, Hilmi Yüksel² \\ 1,2 Department of Business Administration, Dokuz Eylul University, Izmir, Turkey \\ *mahmutsayar@hotmail.com \\ *Orcid No: 0000-0002-1852-6276
}

Received: 20 May 2020

Accepted: 14 September 2020

DOI: 18466/cbayarfbe. 740343

\begin{abstract}
Electricity distribution networks are critical to the delivery of energy and the continuity of the economy. The healthy and efficient operation of these networks depends on the prediction of failures, their early detection and the rapid recovery of the resulting failures. The causes of failure are internal and external factors. Many studies in different sectors that use different techniques for failure prediction in the literature. The use of artificial intelligence techniques, which are becoming increasingly important today, in failure estimates; in terms of estimation success and effectiveness, it brings many privileges compared to other techniques. In this study, a status prediction model has been developed by using artificial neural network (ANN) technique for power outages and healthy working conditions of the electricity distribution network installed in Salihli district of Manisa province. In previous studies, using artificial intelligence techniques in the energy sector generally focused on one component of network, lifetime, energy demand estimation, battery life and goods failures. The effect of meteorological factors has not been studied on the distribution network situation using artificial intelligence techniques. In this study we use hourly power outages and hourly meteorological factors that cause failures or healthy conditions. It is aimed to effective risk management and make anticipation of power outage occurring in electricity transmission network, to make preventive maintenance for failures, to make suggestions for early intervention and shortening downtime and maintenance.
\end{abstract}

Keywords: Production and Service Systems, Operations Management, Artificial Intelligence, Electric Power Distribution Network, Fault Diagnosis, Risk Management, Reliability

\section{Introduction}

Artificial intelligence (AI) can be defined as the whole of multidisciplinary theories, techniques, concepts and technologies applied to develop machines that can simulate intelligence. AI is a science that has been researched and developed in many fields such as machine learning (ML), image processing, natural language processing and robotics etc. The success of machine learning algorithms is shaped by the experience preferences of the model developer. For this reason, AI's success in industrial applications depends on the developer's capabilities. AI is a discipline that focuses on developing, validating, and implementing learning algorithms for industrial applications. AI will be integrated with cyber physical systems, Internet of Industrial Things (IIoT), big data and cloud computing in the process of expanding Industry 4.0 integration. 
As a result, industry processes will acquire flexible, efficient and sustainable capabilities [1]. Artificial neural networks (ANN) is one of the artificial intelligence techniques. It can be defined as one of the important modeling tools used for modeling complex problems in many disciplines, which are easier to use recently in line with the developments in hardware and software. ANNs are composed of interconnected adaptable processing elements that can perform parallel calculations [2]. The ANN technique uses what is known about the functionality of biological organisms to solve complex problems. The advantages of ANN models are due to the characteristics of biological systems such as nonlinearity, high parallelism, error tolerance, learning and fuzzy computing privileges [3].

In previous studies, using artificial intelligence techniques in the energy sector generally focused on issues such as energy demand estimation, battery life, battery charging time, wind power plants, lifetime and electronic goods failures. Studies on failures in power distribution network are generally on one component of the network. The effect of meteorological factors has not been studied on the distribution network situation using artificial intelligence techniques. Factors causing failure in electricity distribution networks are separated into two groups, internal and external factors. Internal factors include transmission cable length, transformer properties, equipment, brands, etc. external factors include meteorological factors. In this study, it is aimed to create a real-time failure and healthy conditions prediction system of the electric power distribution network in dynamic environment conditions, to increase the security level of the system and to reduce system failure probabilities using ANN. By using historical, real time and predictive meteorology data and healthy/faulty status data; system faults and healthy conditions have been estimated in Salihli district.

\section{Artificial Neural Network (ANN)}

Artificial neural networks have been developed based on the working principle of the brain's nerve structure. As it is known, the learning event of the brain takes place through experiments. It consists of three main sections: input layer, hidden layer and output layer. The first entries of the input data into the neural network are done through the input layer. The values in this layer are moved to the neural network and neurons transmit information to the other layer as value. The hidden layer is located between the input and output layers. In this layer, the depth and number of neurons are done by experimental studies. The output values of the network are created in the output layer [4].

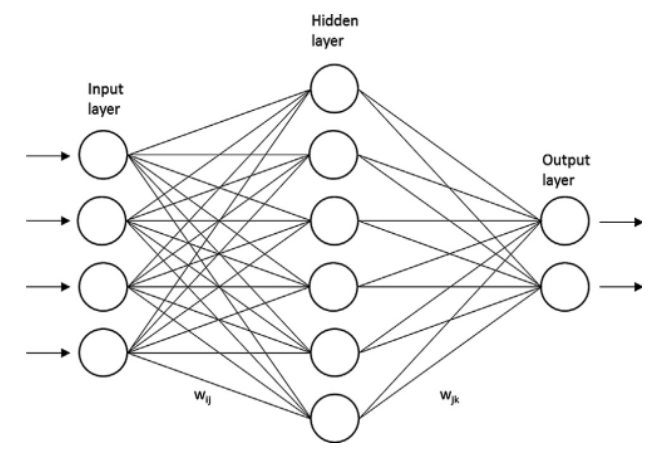

Fig. 1. General Structure of an Artificial Neural Network [5].

ANN is defined as a model consisting of one or more layers formed by neurons and calculating based on the working principle of the human brain. With the selection of the calculation type, it is decided that the ANN model will have feedforward or back propagation architecture. In the ANN learning process, weights of neural network connections are adjusted according to learning rules. Neural networks can be designed according to supervised learning, unsupervised learning and reinforcement learning method. While deciding which of these learning methods to use, it is taken into consideration the problem studied. Activation functions such as hyperbolic tangent, sigmoid, radial basic function and Rectified Linear Unit are commonly used in ANN [6]. The multi-layer perceptron (MLP) consists of the input layer, one-multiple hidden layers and output layers. Each neuron in one layer is connected with every node in the next layer. MLP uses back propagation method in supervised learning situations [7].

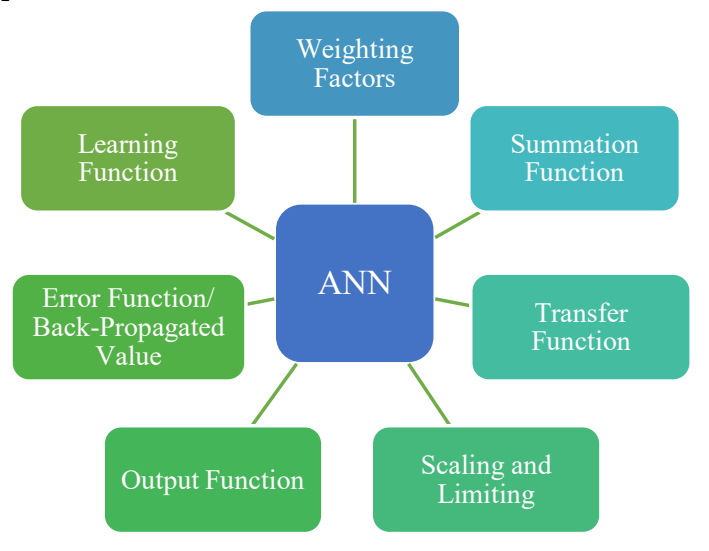

Fig. 2. Major Components of an Artificial Neuron [8]. 
ANN, developed by inspiring the brain's nerve structure. The brain performs the basic learning event with the data obtained from the experiences. This bioinspired method will have an important place in the computer and computing industry. ANN is different from traditional computing and it does not use traditional programming methods. In solving the problems, it generally generates parallel networks and focuses on the training of these networks. ANN includes processes such as behaving, reacting, selforganizing, learning, generalizing, and forgetting [8].



Fig. 3. A Basic Artificial Neuron [8].

In Fig. 3, x(n) shows the various inputs to the network. Each of these input values is multiplied by the link weights indicated mathematically with $\mathrm{w}(\mathrm{n})$. The results of the products are summed or subjected to other mathematical calculations. Output is produced after processing with a transfer function to produce results [8].

When calculating the output of the hidden layer, the $\omega_{i j}$ connection weight and the bias $b_{i}$ between the hidden layer and the previous layer are used:

$$
H_{j}=f\left(\sum_{i=1}^{n} \omega_{i j} x_{i}+b_{i}\right), j=1,2, \ldots . l
$$

In the formula, $l$ represents the number of hidden layer neurons and $f$ represents the activation function of the hidden layer. The final output of the ANN is calculated as follows:

$$
O_{k}=f\left(\sum_{j=1}^{n} \omega_{i j} H_{J}+b_{j}\right), k=1,2, \ldots . m
$$

Where $m$ represents the number of neurons in the output layer, $\omega_{i j}$ is connection weight and $b_{j}$ is bias between the output layer and hidden layer.

The Mean Relative Error Rate (MRER) is determined as the error function of the $\mathrm{SOH}$ estimation model. Mean Absolute Error (MAE) was used as an error function in the training process of the RUL estimation model. The formulas of MRER and MAE are as follows:

$$
\begin{gathered}
\text { MRER }=1 / m \sum_{k=1}^{m}\left|\left(Y_{k}-O_{k}\right) / Y_{k}\right|, k=1,2, \ldots . m \\
M A E=1 / m \sum_{k=1}^{m}\left|\left(Y_{k}-O_{k}\right)\right|, k=1,2, \ldots . m
\end{gathered}
$$

In the formula, $m$ indicates the number of observations. $Y_{k}$ represents the actual value and $O_{k}$ represents the estimated value of ANN. The formula of the back propagation algorithm used for trainining process as follows:

$$
\Delta \omega_{j k}=-\gamma \frac{\partial e_{k}}{\partial \omega_{j k}}, j=1,2, \ldots . l ; k=1,2, \ldots . m
$$

$\gamma$ indicates the connection weight that takes values in the range of 0-1. It shows the degree of adjustment of the connection weight and bias [9].

ANN is used as alternatives to traditional statistical modeling techniques in different disciplines. Gardner and Dorling provides a general discussion for the final applications of the multilayered perceptron in atmospheric sciences, which is a kind of ANN [10]. Neural networks are used for solving problems related to many areas such as forecasting, optimization, clustering, pattern classification, function approximation, control etc [3], [5]. 


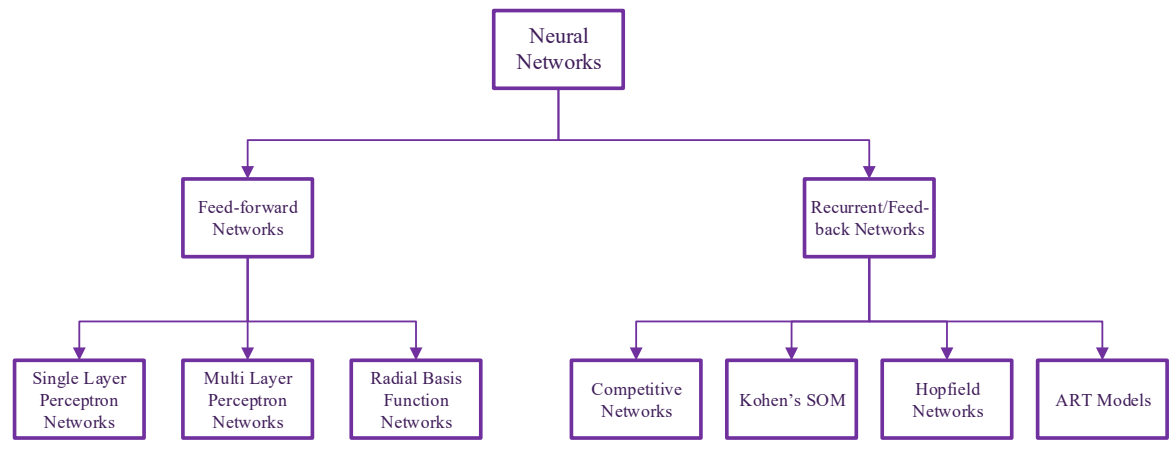

Fig. 4. A Classification of Neural Network Architectures [10].

Learning process and testing process in ANNs are two important steps. After training process in $\mathrm{ANN}$, the success of training process is tested through test data. The success of learning is determined by comparing the predicted values of the training model with the data realized. The working scheme of the ANN is presented in Fig. 5.

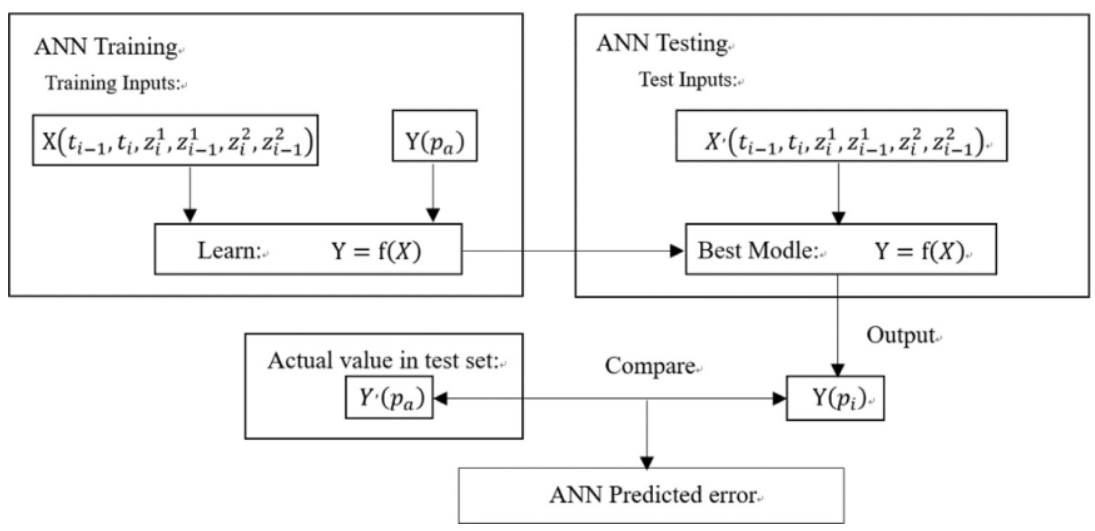

Fig. 5. Artificial neural network scheme used for predictive failure [11].

\subsection{The Multilayer Perceptron Application}

Models consisting of interconnected neurons that show a nonlinear network between the input and output vectors are defined as multilayer perceptron (MLP). Neurons are connected by definite weights and output values produced. Signals and weights are a function of the sum of the inputs to the neuron replaced by the activation function. It is formed by combining many nonlinear transfer functions that enable the multilayer perceptron to converge to nonlinear functions.

Since the logistic function can be easily derived, it is widely used in transfer functions. The output of a neuron is determined by the connection weight and this output is transmitted to the neuron of the next layer. With this structure, a multilayered sensor is known as feed forward neural network. The structure of a multilayer perceptron varies depending on the situation. When the input layer of neural networks (NN) is examined, no calculation is made in this layer. The task of this layer is to ensure that the input vector is transferred to the neural network. A multilayered perceptron consists of one or more hidden layer. It has only one output layer. MLP are defined as fully connected to each neurons, depending on each node in the previous and next layer [10]. Each layer except the input layer takes the necessary actions using the output from the previous layer and transfers the results to the next layer [12].

Multilayered perceptron gain the ability to learn using training data set consisting of input and relevant outputs. During the training process, the appropriate amount of training data is given to the multilayer perceptron and the weights in the network are adjusted 
until the desired input-output level is achieved. MLP are trained by supervised learning method. During training process, the output of the MLP for a given input may differ from the desired output. This error is defined as the difference between the actual and expected value. General error is reduced by using the value of the error signal to determine the values of connection weights in the network during training. The training of a MLP is done through trials where certain connection weights are re-determined to resolve the network modeling relationship. Many algorithms that can be used to train a multi-layered perception. The backpropagation algorithm uses this method to find the general min value of the error surface [10].

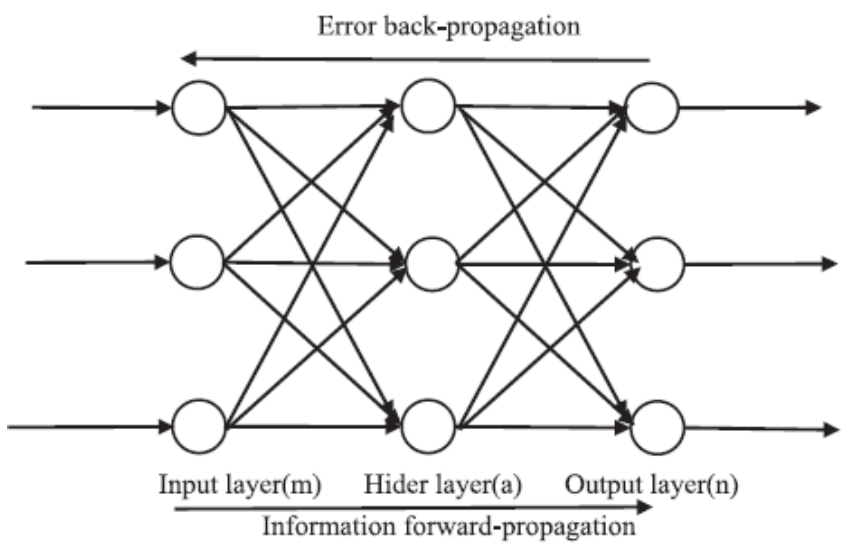

Fig. 6. Structure diagram of a three-layer back propagated neural network (BPNN) [13].

The back propagation algorithm used by Rumelhalt et al is the most suitable method for the training of the MLP. Connection weights in neural network are initially determined as small random values. After the initial calculations, the backpropagation method find out the local gradient of the error surface. It updates the weights in the steepest local gradient direction. It is hoped that the weights will approach the global minimum of the error surface. Training must be finished when the multi-layer perceptron's performance on independent test data is maximized and network error is minimized [10].

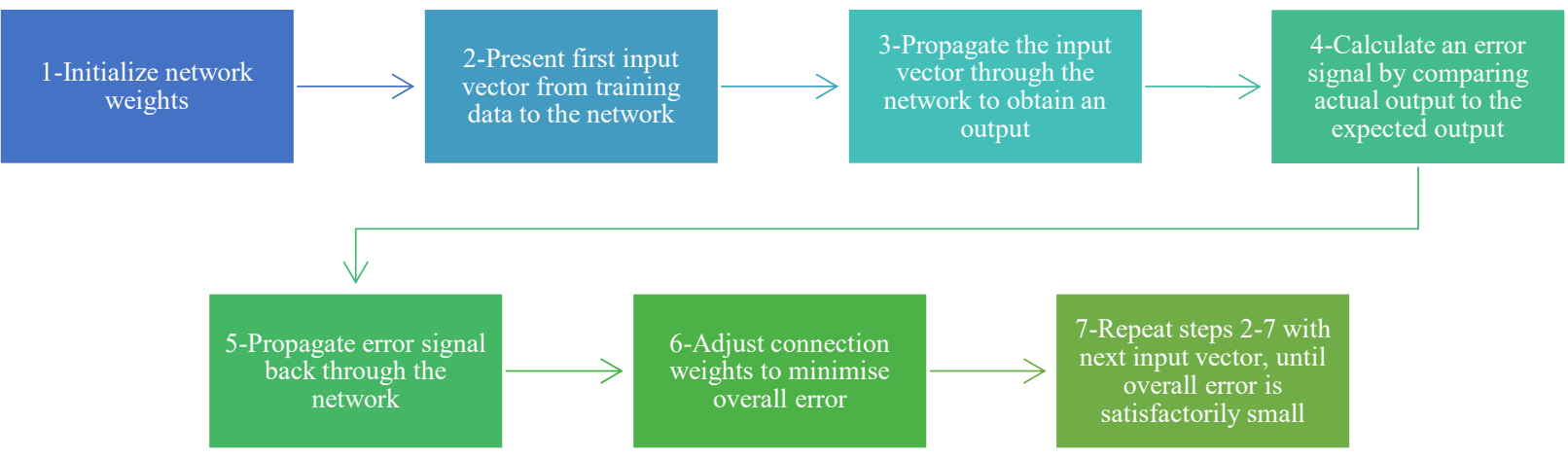

Fig. 7. The back propagation algorithm [10].

\subsection{Teaching an Artificial Neural Network}

Training an ANN is similar to the learning method of the brain. The brain learns through experiences and experiments. In ANNs, there are three learning rules under reinforcement learning (RL), supervised learning (SL) and unsupervised (USL) learning.
In the SL method, the real output of a NN is compared with the desired output. The randomly assigned connection weights are then set by the network. In this way, the next iteration generates a closer match between actual and desired output. While the learning method is running, it tries to minimize valid errors of all processing items. This global error reduction is 
constantly changing the connection weights until an acceptable accuracy value is achieved over time. The training data set provides input and output data to the neural network that occurred in previous times. The corresponding desired output set is also provided for each input set. Actual data is used in most applications. The learning process can take a long time with the hardware that has inadequate processing power. The training of neural networks is completed when it reaches the level of predefined parameters. When training is complete, the current weights are often frozen. For the success of the neural networks calculation, the training data set must be in a large volume containing all the necessary information [8].

Unsupervised learning is an important area where great advances in the future will be made. It paves the way for computers to learn robotically on their own in the future. Such networks are not widely used. These networks do not use any external factors to update their connection weights. Instead, it monitors its performances internally. These networks examine trends or regularity in the input signals and adapt them to the network. The network has some information about how to organize itself. This information is included in learning rules and network architecture. In the training of clusters, weights of only the winning process elements are updated with the learning competition rule [8].

The learning rate is closely related to the learning performance of the network in ANN. The learning rate is the value that indicates how important we consider the error. Given the high rate of learning values errors lead to big changes. Choosing a high learning rate reduces the learning ability of the network. Even with small values, it extend the learning time of the ANN. Therefore, the optimal value should be chosen.

\section{Electric Power Distribution}

Electrical energy makes our life easier in almost every field. It is also a very important component for the development of the industry. Electricity is generally derived from fossil fuels, nuclear and other nonrenewable sources. New power generation systems can easily fail due to random features and effects. For this reason, there are many studies on the reliability and stability problem of the system. System reliability can generally be defined as the probability of reaching certain targets at the desired level under certain internal and external factors. Reliability engineering started to develop after 1940 with the development of statistical methods. This engineering field has started to be used frequently in defense industry. In the 1960s, electrical errors and other system instabilities began to appear in many countries such as Japan and the USA. Power system reliability has been emphasized in the literature since these dates. The power system consists of three main parts. The generation system, transmission system and distribution system are interconnected to each other. The generation system is separated into two classes as renewable and traditional electricity generation system [14].

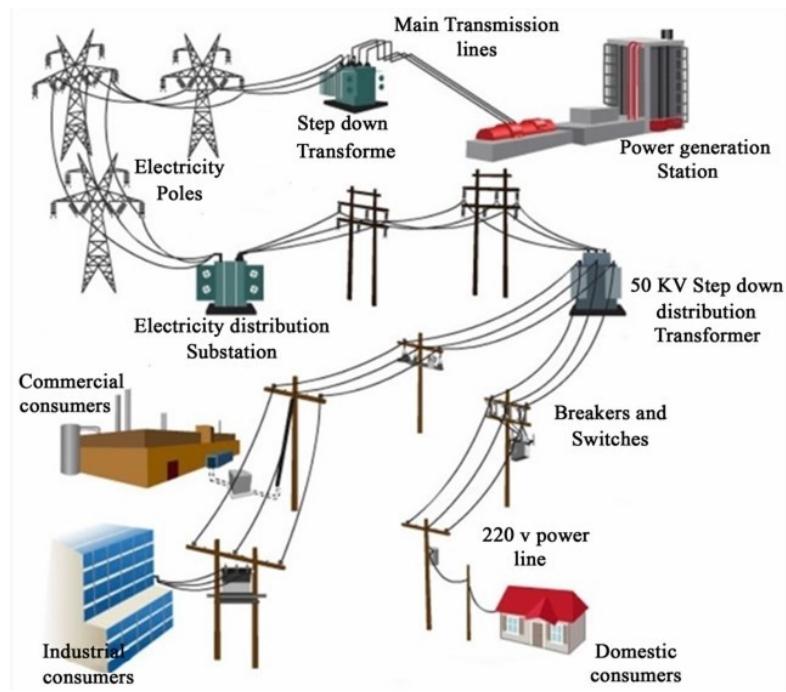

Fig. 8. Electric Power Distribution Network [15].

Transmission lines connect power plants and distribution system. The main objective of the generation and transmission system is to provide sufficient electric power to each main load points within the network. Short circuits or other serious defects often cause the electrical failures in the transmission system. Reliability criteria in the power system are basically the probability of failure, expected reduced load and the expected load reduction time. Indices evaluating the distribution system reliability are used as customer average interrupt frequency index (CAIFI), customer average interrupt duration index (CAIDI), system average interrupt frequency index (SAIFI), system average interrupt duration index (SAIDI). Production, transmission and distribution system will work uninterruptedly in all working situations. Therefore, handling three system reliability simultaneously is an important approach to increase overall electric power system reliability [14]. 
The success of failure diagnostics plays a crucial role in the reliable and healthy operation of power supplies. The applications of expert systems on failure diagnostics give successful results. Besides, application times take a long time [16].

Yongxing et al. calculated the probability of power network failure considering their some meteorological factors. Calculating the probability of real time failure of electric power transmission lines is significant in terms of online risk assessment and real time reliability of the power system network. In the study, fuzzy member functions of the factors were examined first. The uncertainty theory is combined with the fuzzy evaluation method to designate the weighting coefficient of layers. Actual data was collected for simulating the model. The model was verified with actual weather data and power network fault data. Research results showed that the recommended method was successful. Mechanical faults are caused by metal damage, wire fracture, insulator chain fracture or crack, foundation collapse, etc. Current studies on electric power network failure mainly includes weather and operational risk assessment. In the study, real-time failure probability assessment method is presented for overhead electric power transmission lines using meteorological factors [17].

McElroy detected four single-phase EHV transformer faults at the American public institution caused by transformer winding resonance. In previous researches, only the errors of the transformer components were detected with the help of statistical analysis methods. Detailed root causes related to faults have not been investigated. In McElroy's study, not only defining the fault mode, but also the main causes of failures are investigated [18]. Coughlin emphasized that there is no scientific research of the relationship between the physical effects and quantitative analysis used in climate change and energy planning studies in the literature. The study includes determinations on how to use climate models to define how these risks can change over time, including the sensitivity of the electrical system to physical weather risk and the elimination of uncertainties [19].

\subsection{Previous Studies Using Artificial Neural Network}

Baqqar uses generalized regression neural network (GRNN), back propagated ANN (BPNN) and adaptive network-based fuzzy inference system (ANFIS) for status monitoring of gearboxes in electric motors [20]. Cristaldi et al. [21] used ANN and statistical approaches to predict the remaining useful lifes of medium and high voltage circuit breakers. Seidgar et al. [22] used a multi-purpose algorithm improved with neural networks for the maintenance schedule and work order to reduce production disruptions [23]. Zhang et al. used methods of ANN and partial incremental capacity to predict the health and remaining useful lifes of the batteries under constant current discharge. Estimations with high reliability and accuracy were made with the proposed methods [9]. Marugán et al. provide a comprehensive review of ANNs used in wind energy systems. Researchers determine the most used methods for applications and shows that ANNs can be an alternative to traditional methods in many cases. According to the literature review in the study, the main difficulties and technological inadequacies regarding the application of ANN to wind turbines are explained [5].

Mousavian et al. proposes a $\mathrm{NN}$ based protection method by investigating the risks against cyber-attacks associated with the data of electric power system transmission. The proposed model can monitor the output of power flow calculations and detect data abnormalities in real time. In the study, it was mentioned that cyber attacks can be as dangerous as physical attacks on the power grid due to the great damage and losses [24]. Silva et al. used an incremental learning algorithm based on real data to predict high impedance failures in electrical power distribution system. The efficiency of recommended evolutionary system to detect and classify failures was compared with the multilayered NN, probabilistic NN and support vector machine(SVM) methods. The results of the study state that the recommended system is suitable for changes [25]. Significant progress is being made in the field of electric power systems in all sectors. Renewable energy sources, their increasing influence, increasing the number of customers and appropriate green energy policies trigger the development of the electrical system. In the light of these developments, Saviozzi et al. used ANN to increase the functionality of the load estimation and load modeling processes required by distribution management systems [26].

Đozić et al. analyze it by developing a model of ANNs to predict $\mathrm{CO}_{2}$ emissions by 2050 based on the EU's current energy policy. With the study, ANN has been effective in predicting the behavior of $\mathrm{CO}_{2}$ emissions. It 
will also change the indicator's value by 2050 to ensure that $\mathrm{CO}_{2}$ emissions are reduced in a planned manner, ensuring timely improvement of economic and energy strategy [27]. Khwaja et al. use ANN based ensemble ML to improve short term electrical charge estimation. The recommended technique combines both strengthening and bagging to train ANN. A group of multiple ANN models were trained in parallel. To obtain the last estimated load, the estimated loads of these models are averaged [28]. The first researches on wind speed estimation were generally done using numerical and physical mathematical models [29]. Lawan et al. proposed a wind condition-forecasting model using topographic machine learning to study wind energy potential in Sibu. In the study where a land-based ANN was developed, it was found that wind speed values can be successfully estimated in the areas where the model is applied [30].

Condition based maintenance method (CBM) predicts a maintenance plan depends on data obtained in case of failure and non-faulty operating situations. Various sensors and monitoring devices such as acousticultrasonic sensor, accelerometer, current measurements and thermocouples are needed to monitor the status of a system with CBM. In addition, it provides highly useful information from environment condition's data such as pressure, humidity, temperature et.al to increase the effectiveness of the model. In the light of this data, certain KPIs are analyzed to reveal potential tendencies that can lead to a critical failure. The aim is to adopt a suitable CBM system that provides forecasting and monitoring capabilities by simultaneously combining important data to ensure maximum reliability [23]. The purpose of Mohamed and Rao's study is to develop a fault diagnostic system for distribution networks using
ANN. In this study, multi-layer feed forward neural network with back propagation algorithm was used. In the study, the determination of the error, identification of the defective parts and the classification of the errors according to the types were carried out [16].

\section{Prediction of Electric Power Distribution Condition Using Artificial Neural Network}

In this section, a case study of the electricity distribution network of Salihli district is presented. The data of the district were obtained from the electricity distribution company and Turkish State Meteorological Service. ANN model was developed for the status of the electricity distribution network by matching the data obtained from these institutions. Learning for the unplanned power outages and healthy conditions of the distribution network has been made and the predictions of the future system status have been made.

\subsection{Data}

Salihli, located in Turkey's Aegean province of Manisa is one of the districts. The ancient city of Sardes, where the first money was used in history and now included in the UNESCO World Heritage Temporary List, is located in this district. The surface area of the district is $1359 \mathrm{~km}^{2}$ and the altitude is $108 \mathrm{~m}$. According to 2019 data, its population is 162787 people. Totally more than 5000 unplanned power cuts occurred in the district in 2017 and 2018. Power outages negatively affects the economic and social life in the district. In our study, the hourly condition (outage or healthy) of the electricity distribution line of Salihli and meteorological data that occurred at that time were used.

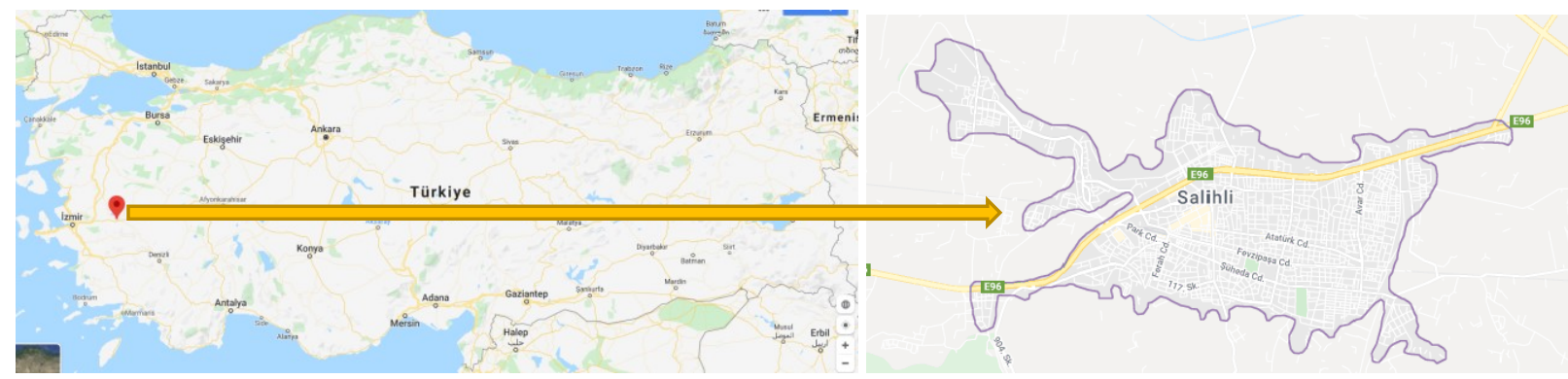

Fig. 9. The location of Salihli in Turkey. 
In this study, we used a total of 11428 hours data set regarding meteorological data and power grid status (1:Outage - 0:Healthy). We randomly divided $80 \%$ (9149) of the data set into learning (training) and 20\% (2279) as test data. We took atmospheric pressure
$(\mathrm{hPa})$, relative humidity $(\%)$, wind speed $(\mathrm{m} / \mathrm{s})$, wind direction $\left({ }^{\circ}\right)$, temperature $\left({ }^{\circ} \mathrm{C}\right)$ and amount of precipitation $\left(\mathrm{mm}=\mathrm{kg} / \mathrm{m}^{2}\right)$ as inputs of the model. Example of data set prepared for training process is presented in Table 1.

Table 1. Part of The Data Set Ready for The Training Process.

\begin{tabular}{|c|c|c|c|c|c|c|c|}
\hline Date \& Hour & $\begin{array}{c}\text { Power } \\
\text { Outage } \\
(1-0)\end{array}$ & $\begin{array}{c}\text { Atmospheric } \\
\text { Pressure }\end{array}$ & $\begin{array}{c}\text { Relative } \\
\text { Humidity } \\
\text { (\%) }\end{array}$ & $\begin{array}{l}\text { Wind Speed } \\
\quad(m \div s n)\end{array}$ & $\begin{array}{c}\text { Wind } \\
\text { Direction }\left({ }^{\circ}\right)\end{array}$ & $\begin{array}{c}\text { Temperature } \\
\left({ }^{\circ} \mathrm{C}\right)\end{array}$ & $\begin{array}{c}\text { Amount of } \\
\text { Precipitation } \\
\left(\mathrm{mm}=\mathrm{kg} \div \mathrm{m}^{2}\right)\end{array}$ \\
\hline $17-1-2018 \quad 13$ & 0 & 993.3 & 46 & 1.6 & 146 & 16.5 & 0 \\
\hline $17-1-2018 \quad 14$ & 1 & 992.4 & 49 & 2.3 & 138 & 15.8 & 0.2 \\
\hline $17-1-2018 \quad 15$ & 0 & 991.6 & 48 & 2.7 & 196 & 15.4 & 0 \\
\hline $17-1-2018 \quad 16$ & 0 & 990.7 & 49 & 4 & 212 & 15.5 & 0.4 \\
\hline $17-1-2018 \quad 17$ & 1 & 989.8 & 55 & 2.4 & 138 & 13.6 & 0 \\
\hline $17-1-2018 \quad 18$ & 1 & 989 & 57 & 2.6 & 267 & 14.4 & 0 \\
\hline $17-1-201819$ & 0 & 988.7 & 57 & 1.9 & 38 & 15 & 0 \\
\hline $17-1-201820$ & 0 & 988 & 57 & 1.5 & 299 & 16 & 0.2 \\
\hline $17-1-201821$ & 1 & 987.4 & 64 & 1.8 & 88 & 13.9 & 0 \\
\hline $17-1-201822$ & 0 & 986.4 & 68 & 1.8 & 104 & 13.4 & 0 \\
\hline$\ldots \ldots$ & $\ldots \ldots$ & $\ldots \ldots$ & ..... & $\ldots \ldots$ & $\ldots \ldots$ & $\ldots \ldots$ & $\ldots \ldots$ \\
\hline \multicolumn{8}{|c|}{ Total 11428 hours of data set } \\
\hline
\end{tabular}

General statistical distribution of input data are presented as Fig. 10, Fig. 11, Fig. 12, Fig. 13, Fig. 14 and Fig. 15.

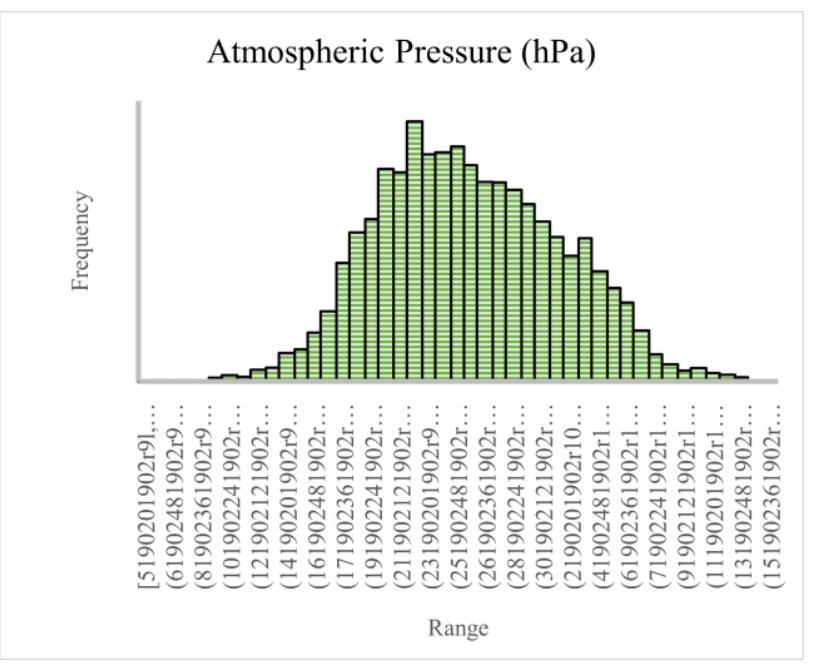

Fig. 10. Atmospheric Pressure Diagram (hPa).

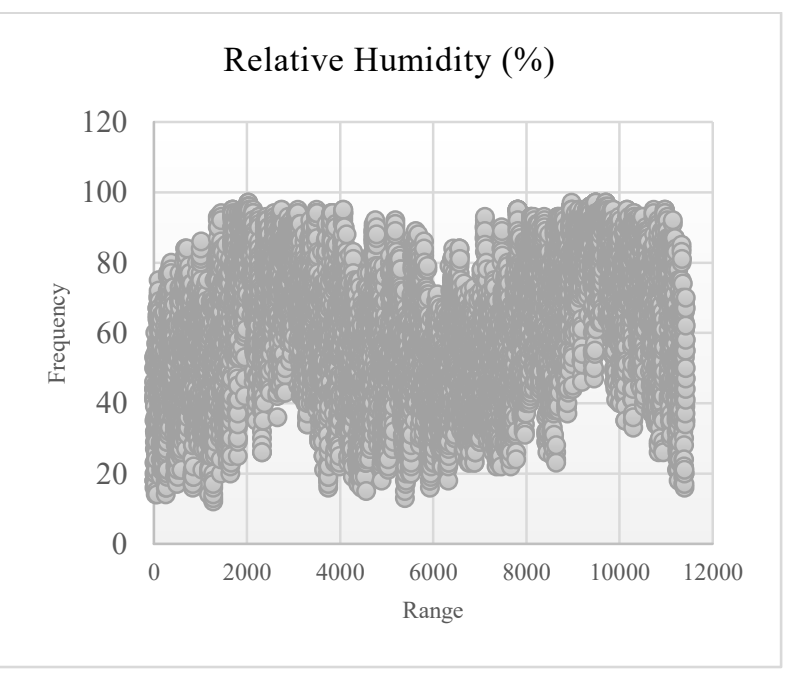

Fig. 11. Relative Humidity Diagram (\%). 


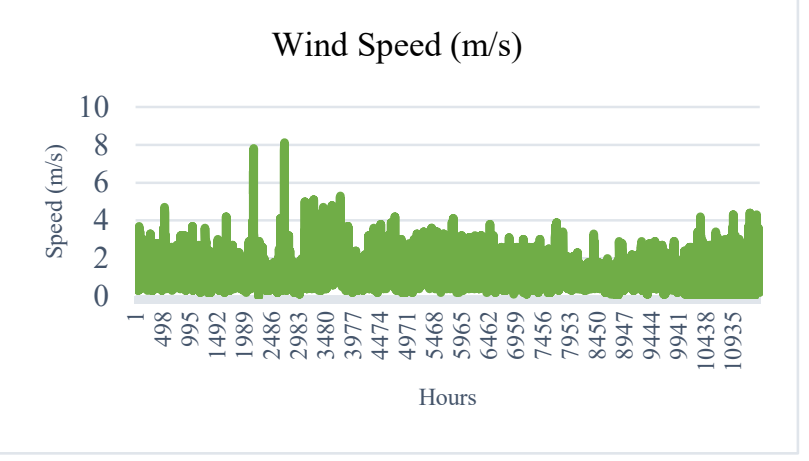

Fig. 12. Wind Speed Diagram.

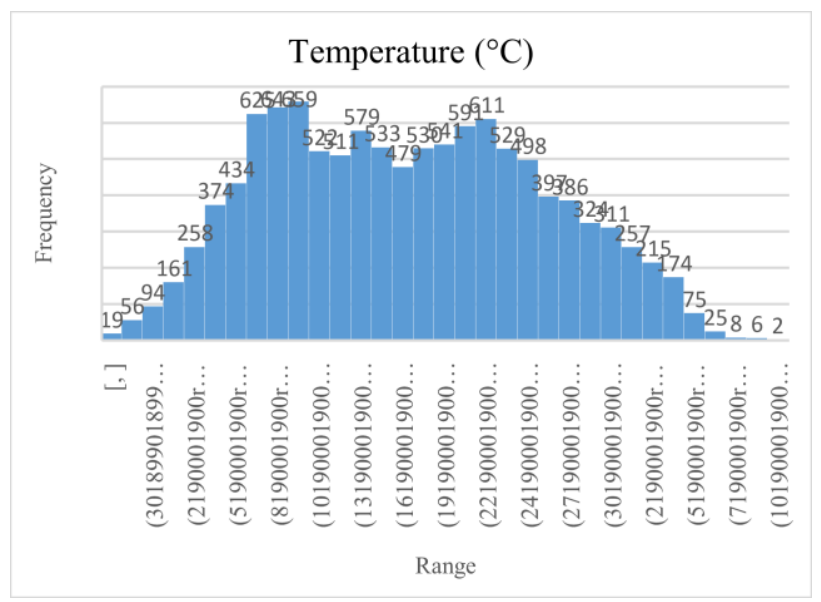

Fig. 14. Temperature Diagram.

As the target of the study, we determined the hourly forecast of the healthy status and power outage conditions of the electricity distribution networks.

\subsection{ANN Model}

In this study, the situation of the network is tried to be estimated with the supervised learning method by using meteorological factors, which are the external factor for distribution networks, in cases where the electricity

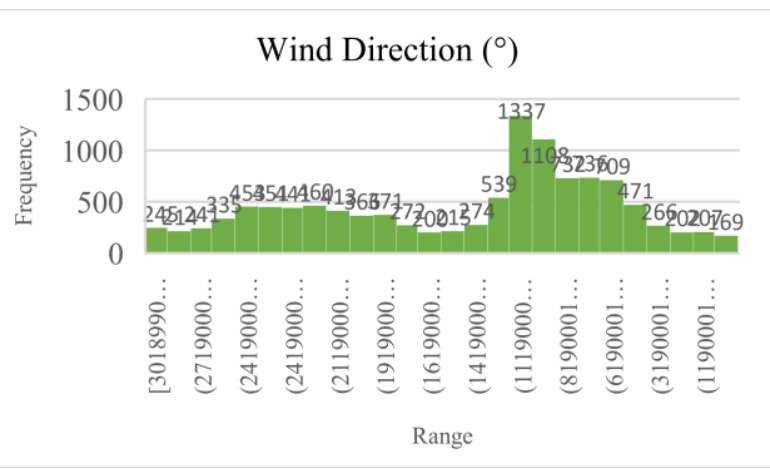

Fig. 13. Wind Direction Diagram.

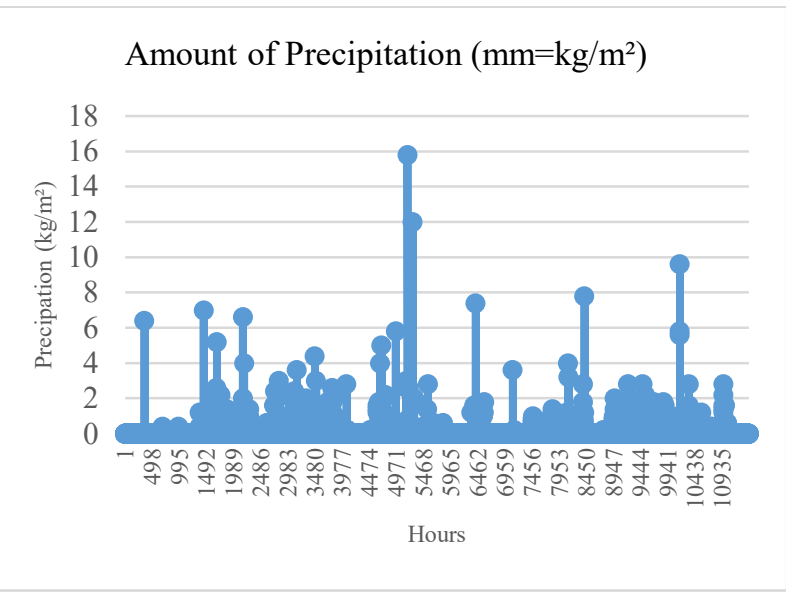

Fig. 15. Amount of Precipitation Diagram.

distribution network is healthy or outage. We established our ANN model after the data cleaning and combining processes. The installation parameters of the model are determined as follows Table 2. The process of cleaning, editing, merging and adapting the data sets from different data sources are very important for the success of the model of ANN. ANN modeling process is shown in Fig. 16 as algorithm steps.

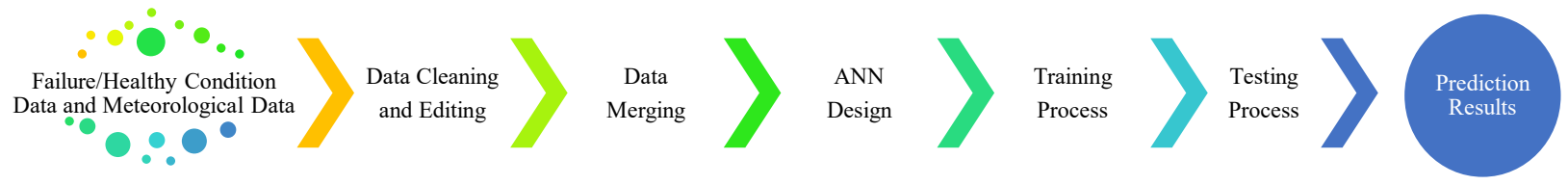

Fig. 16. ANN Design Procedure. 
We set the input parameters as temperature, relative humidity, atmospheric pressure, wind speed, wind direction and amount of precipitation and we determined the neural network as multilayer perceptron (MLP). We tried different network structures with the SPSS Modeler in determining the appropriate model. For this, we have repeatedly updated the model's layers and the number of neurons. As a result of these updates, the neural network structure in Fig. 17 gave appropriate results. Our neural network consists of 12 neurons in the first hidden layer and 9 neurons in the second hidden layer. We set the model type as classification and train neural network 750 times with learning data set. During this learning process, the network learned 9149 hours of data set in total 11428 hours of data set.

Table 2. Parameters of ANN Model.

\begin{tabular}{|c|c|c|}
\hline \multicolumn{3}{|c|}{ Fields } \\
\hline & & Power Grid Status (1:Outage - 0:Healthy) \\
\hline & Predictors(Inputs) & \\
\hline & & Relative Humidity (\%) \\
\hline & & Wind Speed $(\mathrm{m} / \mathrm{s})$ \\
\hline & & Amount of Precipitation $\left(\mathrm{mm}=\mathrm{kg} / \mathrm{m}^{2}\right)$ \\
\hline & & Atmospheric Pressure (hPa) \\
\hline & & Wind Direction $\left(^{\circ}\right)$ \\
\hline & & Temperature $\left({ }^{\circ} \mathrm{C}\right)$ \\
\hline \multicolumn{3}{|l|}{ Build Options } \\
\hline & Basics & \\
\hline & & Neural network model: Multilayer Perceptron (MLP) \\
\hline & & Hidden Layer-1: 12 \\
\hline & & Hidden Layer-2: 9 \\
\hline & Stopping Rules & \\
\hline & & Use max training time (per component model): True \\
\hline & & Customize number of max training cycles: True \\
\hline & & Maximum number of cycles: 750 \\
\hline & & Accuracy (\%): 95.0 \\
\hline & Advanced & \\
\hline & & Overfit prevention set(\%): 2 \\
\hline & & Replicate Results: True \\
\hline & & Random seed: 75987945 \\
\hline & & Missing values in predictors: Delete listwise \\
\hline \multicolumn{3}{|l|}{ Training Summary } \\
\hline & Method & Neural Networks \\
\hline & Records used in training & 9149 \\
\hline & Model type & Classification \\
\hline & Predictors used in model & \\
\hline & & Amount of Precipitation $\left(\mathrm{mm}=\mathrm{kg} / \mathrm{m}^{2}\right)$ \\
\hline & & Atmospheric Pressure $(\mathrm{hPa})$ \\
\hline & & Relative Humidity (\%) \\
\hline & & Temperature $\left({ }^{\circ} \mathrm{C}\right)$ \\
\hline & & Wind Direction $\left({ }^{\circ}\right)$ \\
\hline & & Wind Speed $(\mathrm{m} / \mathrm{s})$ \\
\hline
\end{tabular}




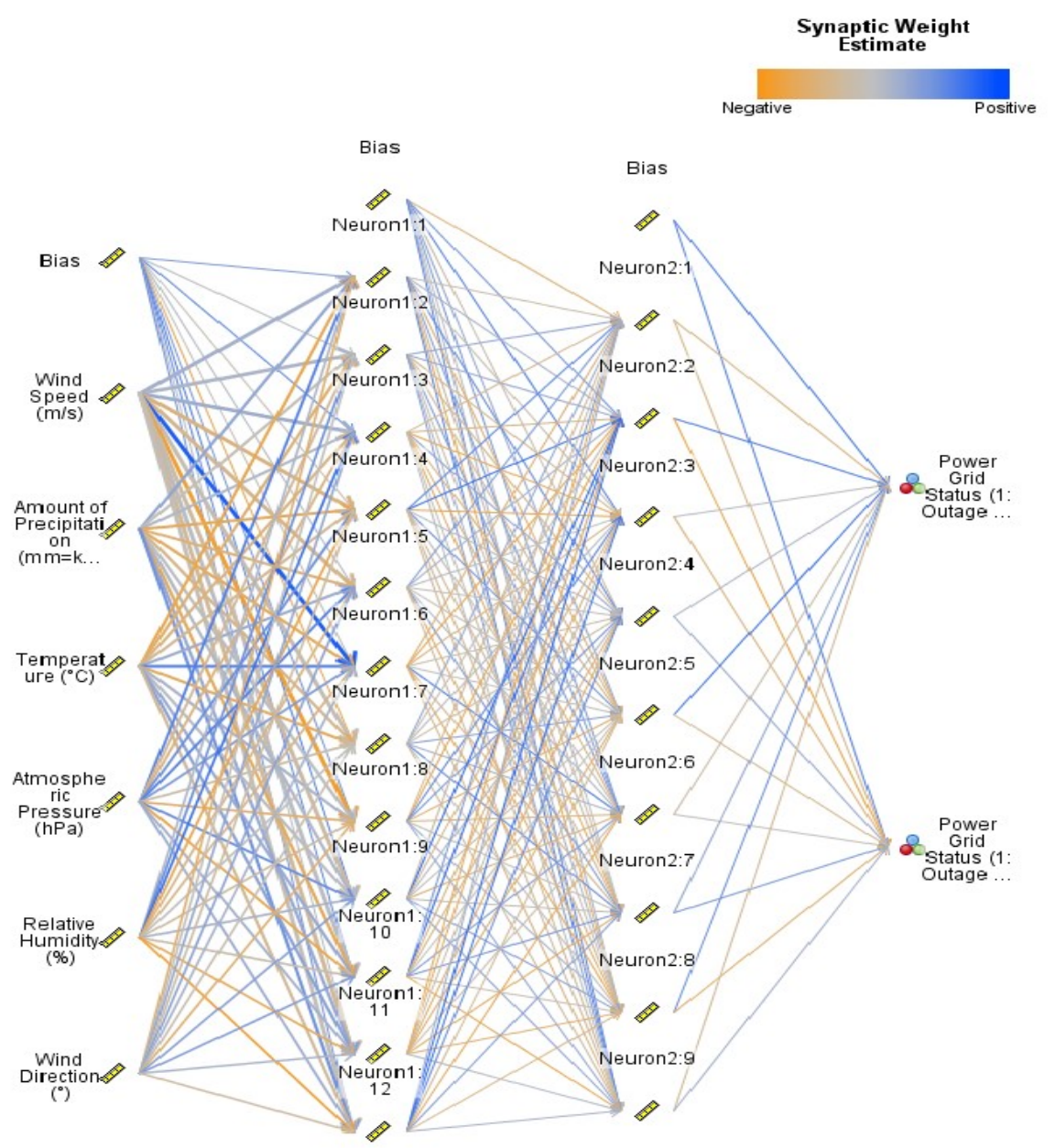

Fig. 17. Artificial Neural Network Model of Salihli Electricity Distribution Network.

Table 3. Actual and Prediction Results of Power Grid Status With Test Data Set (1:Outage - 0:Healthy).

\begin{tabular}{c|cccc} 
Result No & $\begin{array}{c}\text { Power Grid Status } \\
\text { (1:Outage - 0:Healthy) }\end{array}$ & $\begin{array}{c}\text { Prediction of Power Grid Status } \\
\text { (1:Outage - 0:Healthy) }\end{array}$ & \\
\cline { 1 - 3 } 1 & 0 & 0 & True Positive \\
2 & 1 & 0 & False Negative \\
3 & 0 & 0 & \\
4 & 1 & 0 & \\
5 & 1 & 0 & \\
6 & 0 & 0 & \\
7 & 0 & 0 & \\
8 & 1 & 1 & True Negative \\
9 & 1 & 1 & \\
10 & 0 & 0 & \\
11 & 1 & 0 & \\
12 & 0 & 0 & \\
13 & 1 & 0 & \\
14 & 0 & 1 & \\
15 & 1 & $\ldots$ & \\
$\ldots$ & $\ldots$ & $\ldots$ & \\
$\ldots$ & $\ldots$ & 0 & \\
2278 & 0 & & \\
\end{tabular}




\section{Results and Discussion}

The ANN model is runned 750 times using the learning dataset of $9149(80 \%)$ hours and the test dataset of 2278 $(20 \%)$ hours, which is randomly partitioned into two parts over the data set of Salihli district. The results of the prediction of the model are given in confusion matrix (Table 3 and Table 4).

After the learning process, using the 2278 hour test data set, in the prediction process regarding the status of the network; 1491 healthy conditions (True Positive) and 117 fault conditions (True Negative) were estimated correctly.

Table 4. Classification for Power Grid Status With Test Data Set (1:Outage - 0:Healthy).

\begin{tabular}{c|ccc} 
& \multicolumn{3}{c}{ Predicted } \\
\hline Observed & 0 & 1 & Total \\
\hline 0 & 1491 & 155 & 1646 \\
1 & 515 & 117 & 632 \\
Total & 2006 & 272 & 2278
\end{tabular}

In 155 cases, there was no real fault, but the result of a failure situation was estimated (False Positive). It has been estimated that the network will operate properly for 515 cases where there are actually a failures (False Negative). Overall predictive accuracy of the model is given Table 5. and Fig. 18.
Table 5. Overall Predictive Accuracy of The Model with Test Data.

\begin{tabular}{l|cc} 
& Overall Prediction & $\%$ \\
\hline Correct & 1608 & $70.59 \%$ \\
Wrong & 670 & $29.41 \%$ \\
Total & 2278 & $100.00 \%$
\end{tabular}

According to the results, when all the estimation scores are combined, our model predicts the hourly status of the electricity distribution network of Salihli district by $70.59 \%$ accuracy and $29.41 \%$ error.

Overall Prediction Success \%

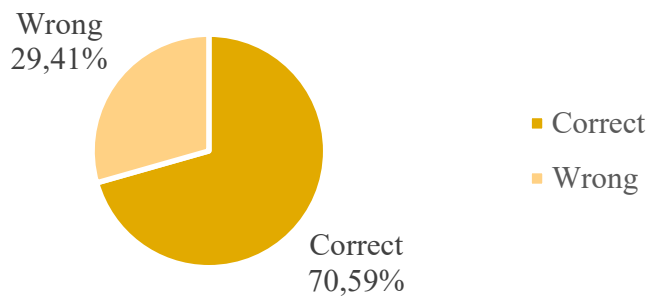

Fig. 18. Overall Prediction Score Chart.

As a result, when all the estimation scores are combined, our model predicts the hourly status of the electricity distribution network of Salihli district by $70.59 \%$ accuracy and $29.41 \%$ error.

Table 6. Predictor Importance of ANN Model.

\begin{tabular}{|c|c|c|}
\hline \multicolumn{3}{|c|}{$\begin{array}{l}\text { Predictor Importance } \\
\text { Target: Power Grid Status }\end{array}$} \\
\hline \multirow[t]{2}{*}{$32 \%$} & $13 \%$ & $9 \%$ \\
\hline & \multicolumn{2}{|c|}{ Predictor Importance (\%) } \\
\hline$\square$ Wind Speed (m/s) & \multicolumn{2}{|c|}{$32 \%$} \\
\hline$\square$ Amount of Precipitation $\left(\mathrm{mm}=\mathrm{kg} / \mathrm{m}^{2}\right)$ & \multicolumn{2}{|c|}{$19 \%$} \\
\hline$\square$ Temperature $\left({ }^{\circ} \mathrm{C}\right)$ & \multicolumn{2}{|c|}{$18 \%$} \\
\hline$\square$ Atmospheric Pressure $(\mathrm{hPa})$ & \multicolumn{2}{|c|}{$13 \%$} \\
\hline$\square$ Relative Humidity (\%) & \multicolumn{2}{|c|}{0} \\
\hline$\square$ Wind Direction $\left(^{\circ}\right)$ & \multicolumn{2}{|c|}{$9 \%$} \\
\hline
\end{tabular}

In this article, where we examine the effect of meteorological factors on the status of electricity distribution network in Salihli district, it is analyzed that the importance of each factor in predicting the status of the network is different. Predictors importance are given in Table 6. In our ANN model, it is calculated 
that the wind speed is the best predictor for the network status for the district of Salihli (32\%). The predictive significance levels of precipitation amount and temperature values are close to each other; The amount of precipitation is $19 \%$ and the temperature is $18 \%$ predictive. Atmospheric pressure is $13 \%$ and relative humidity is $10 \%$ predictive; The wind direction has the lowest predictive significance with a value of $9 \%$.

According to the results, the ANN model can make the forecast with a high success rate of the distribution network status by using the future forecast data of meteorological factors. Real-time data sharing between electricity distribution companies and meteorological measurement institutions is of great importance in terms of electricity distribution network security.

\section{Conclusion}

This study was carried out to draw attention to the need for simultaneous evaluation of internal factors (transformer information, cable lengths, brand ... etc.) and external factors (meteorological) affecting the working status of electricity distribution networks. In our study, ANN model was developed by using only external factors (meteorological) that affect the working status of the electricity distribution network. According to the obtained results, hourly estimation of the healthy and power outage conditions of the network with $70.59 \%$ accuracy has been realized successfully. Thanks to our ANN model, the status of the electricity distribution network of Salihli can be estimated with $70.59 \%$ accuracy level by using meteorological forecast data of the future. It will be appropriate to take preventive precaution by the relevant institutions to reduce the damage caused by wind speed, air temperature and other factors in order of importance to the transmission lines.

In later studies, with the Industry 4.0 infrastructure to be integrated into the electricity distribution networks, the data on the internal (transmission cable lengths, cable cross section, transformer properties, brands, etc) and external factors of the network will be obtained more accurately and easily. In addition, better prediction results can be obtained with deep learning models that will be created by using different learning algorithms and increasing the number of layers, neurons and epoch values in neural networks.

\section{Author's Contributions}

Mahmut Sayar: Drafted and wrote the manuscript, performed the experiment, result analysis and interpretation.

Hilmi Yüksel: Supervised the manuscript's progress, interpretation and helped in manuscript preparation.

\section{Ethics}

There are no ethical issues after the publication of this manuscript.

\section{References}

1. Lee, J., Davari, H., Singh, J. ve Pandhare, V. (2018). Industrial Artificial Intelligence for industry 4.0-based manufacturing systems. Manufacturing Letters, 18, 20-23. doi:10.1016/j.mfglet.2018.09.002.

2. Hecht-Nielsen, R. (1990). Neurocomputing, Addison. Wesely Publishing Company. Hornik, K. Stinchcombe, M. White, H.(1989). Multilayer feedforward networks are universal approximators, Neural Networks, 2(359366), 3168-3176.

3. Basheer, I. A. ve Hajmeer, M. (2000). Artificial neural networks: fundamentals, computing, design, and application. Journal of microbiological methods, 43(1), 3-31.

4. Shaban, S. E., Hazzaa, M. H. ve El-Tayebany, R. A. (2019). Applying Monte Carlo and artificial intelligence techniques for 235U mass prediction in samples with different enrichments. Nuclear Instruments and Methods in Physics Research, Section A: Accelerators, Spectrometers, Detectors and Associated Equipment, 916, 322-326. doi:10.1016/j.nima.2018.10.008.

5. Marugán, A. P., Márquez, F. P. G., Perez, J. M. P. ve RuizHernández, D. (2018). A survey of artificial neural network in wind energy systems. Applied energy, 228, 1822-1836.

6. Kruse, R., Borgelt, C., Braune, C., Mostaghim, S. ve Steinbrecher, M. (2016). Multilayer perceptrons. Computational Intelligence içinde (ss. 47-92). Springer.

7. Zhang, J. ve Li, J. (2020). Testing and verification of neuralnetwork-based safety-critical control software: A systematic literature review. Information and Software Technology, 106296.

8. Anderson, D. ve McNeill, G. (1992). Artificial neural networks technology. Kaman Sciences Corporation, 258(6), 1-83.

9. Zhang, S., Zhai, B., Guo, X., Wang, K., Peng, N. ve Zhang, X. (2019). Synchronous estimation of state of health and remaining useful lifetime for lithium-ion battery using the incremental capacity and artificial neural networks. Journal of Energy Storage, 26, 100951.

10. Gardner, M. W. ve Dorling, S. R. (1998). Artificial neural networks (the multilayer perceptron) - a review of applications in the atmospheric sciences. Atmospheric environment, 32(1415), 2627-2636 
11. Lu, Y., Sun, L., Zhang, X., Feng, F., Kang, J. ve Fu, G. (2018). Condition based maintenance optimization for offshore wind turbine considering opportunities based on neural network approach. Applied Ocean Research, 74, 69-79.

12. Berecibar, M., Devriendt, F., Dubarry, M., Villarreal, I., Omar, N., Verbeke, W. ve Van Mierlo, J. (2016). Online state of health estimation on NMC cells based on predictive analytics. Journal of Power Sources, 320, 239-250.

13. Sun, W. ve Xu, Y. (2016). Financial security evaluation of the electric power industry in China based on a back propagation neural network optimized by genetic algorithm. Energy, 101, 366-379.

14. Wang, J., Zhao, X., Guo, X. ve Li, B. (2018). Analyzing the research subjects and hot topics of power system reliability through the Web of Science from 1991 to 2015. Renewable and Sustainable Energy Reviews, 82, 700-713. doi:10.1016/j.rser.2017.09.064.

15. Pngio. (2020). Electric Power Distribution. CRC Press. https://pngio.com/images/png-a1356339.html (accessed at $05.17 .2020)$

16. Mohamed, E. A. ve Rao, N. D. (1995). Artificial neural network based fault diagnostic system for electric power distribution feeders. Electric Power Systems Research, 35(1), $1-10$.

17. Yongxing, C., Yufang, Z. ve Hongyu, Z. (2017). Real-time Evaluation Model of Power Line Fault Probability based on Multiple Meteorological factors. Procedia Computer Science, 107, 231-235. doi:10.1016/j.procs.2017.03.084.

18. McElroy, A. J. (1975). On the significance of recent EHV transformer failures involving winding resonance. IEEE Transactions on Power Apparatus and Systems, 94(4), 13011316. doi:10.1109/T-PAS.1975.31968.

19. Coughlin, K. ve Goldman, C. (2008). Physical Impacts of Climate Change on the Western US Electricity System: A Scoping Study. Lawrence Berkeley National Laboratory, 29. http://escholarship.org/uc/item/8rc6q28 adresinden erişildi.

20. Baqqar, M. (2015). Machine Performance and Condition Monitoring Using Motor Operating Parameters Through Artificial Intelligence Techniques. University of Huddersfield.

21. Cristaldi, L., Leone, G., Ottoboni, R., Subbiah, S. ve Turrin, S. (2016). A comparative study on data-driven prognostic approaches using fleet knowledge. 2016 IEEE International
Instrumentation and Measurement Technology Conference Proceedings içinde (ss. 1-6).IEEE.

22. Seidgar, H., Zandieh, M. ve Mahdavi, I. (2017). An efficient meta-heuristic algorithm for scheduling a two-stage assembly flow shop problem with preventive maintenance activities and reliability approach. International Journal of Industrial and Systems Engineering, 26(1), 16-41.

23. Diez-Olivan, A., Del Ser, J., Galar, D. ve Sierra, B. (2019) Data fusion and machine learning for industrial prognosis: Trends and perspectives towards Industry 4.0. Information Fusion, 50, 92-111. doi:10.1016/j.inffus.2018.10.005.

24. Mousavian, S., Valenzuela, J. ve Wang, J. (2013). Real-time data reassurance in electrical power systems based on artificial neural networks. Electric Power Systems Research, 96, 285295.

25. Silva, S., Costa, P., Gouvea, M., Lacerda, A., Alves, F. ve Leite, D. (2018). High impedance fault detection in power distribution systems using wavelet transform and evolving neural network. Electric Power Systems Research, 154, 474483.

26. Saviozzi, M., Massucco, S. ve Silvestro, F. (2019). Implementation of advanced functionalities for Distribution Management Systems: Load forecasting and modeling through Artificial Neural Networks ensembles. Electric Power Systems Research, 167, 230-239. doi:10.1016/j.epsr.2018.10.036.

27. Đozić, D. J. ve Urošević, B. D. G. (2019). Application of artificial neural networks for testing long-term energy policy targets. Energy, 174, 488-496.

28. Khwaja, A. S., Anpalagan, A., Naeem, M. ve Venkatesh, B. (2020). Joint bagged-boosted artificial neural networks: Using ensemble machine learning to improve short-term electricity load forecasting. Electric Power Systems Research, 179, 106080. doi:10.1016/j.epsr.2019.106080.

29. Carta, J. A., Ramirez, P. ve Velazquez, S. (2009). A review of wind speed probability distributions used in wind energy analysis: Case studies in the Canary Islands. Renewable and sustainable energy reviews, 13(5), 933-955.

30. Lawan, S. M., Abidin, W. A. W. Z. ve Masri, T. (2019) Implementation of a topographic artificial neural network wind speed prediction model for assessing onshore wind power potential in Sibu, Sarawak. Egyptian Journal of Remote Sensing and Space Science. doi:10.1016/j.ejrs.2019.08.003. 